Educación Física y Ciencia, vol. 19, n² 2, e036, diciembre 2017. ISSN 2314-2561

Universidad Nacional de La Plata.

Facultad de Humanidades y Ciencias de la Educación.

Departamento de Educación Física

\title{
Espacios Conceptuales de Actuación Profesional: vivencia, práctica y entrenamiento
}

\author{
Conceptual Spaces of the Professional Performance of the Physical \\ Education Teacher: experience, practice and training
}

\author{
Luis Linzmayer Gutiérrez * \\ * Universidad del Bio-Bio, Chile | luislinzmayer@gmail.com
}

\section{PALABRAS CLAVE RESUMEN}

Actuación profesional

Educación física

Dominio conceptual

\section{KEYWORDS}

Professional action

Physical education

Conceptual domain
Los espacios de actuación profesional del profesor de Educación Física (Pérez Gallardo, et.al.2003), aparecen como una forma de diferenciar las responsabilidades y objetivos que el profesional del área debe desarrollar conforme lo indican las características de su profesión. En el presente ensayo se enfatiza que dichos espacios, al conformar en la acción diferentes conceptualizaciones, estos sufren una modificación en su expresión original. De ahí que se reconozcan, en este estudio, como espacios conceptuales de actuación profesional. En su génesis, estos espacios, vinieron a aclarar y diferenciar la complejidad de las intervenciones pedagógicas del profesor de Educación Física. Por ello se indica que, una formación inicial competente, debiera permitir, reconocer y adecuar la acción profesional identificando las variables y los conceptos que cada espacio conceptual implica y que estos no deben confundirse con espacios físicos.

\section{ABSTRACT}

The Performance spaces of the Physical Education Teacher (Pérez Gallardo, et.al.2003) appear to be a way to differentiate the responsibilities and objectives that the professional area should develop as indicated by the characteristics of their profession. In this work, it is emphasized that these spaces, while forming different conceptualizations in action, suffer a modification in its original expression. That's why, they are recognized, in this study, as conceptual spaces of performance. In their genesis, these spaces came to clarify and differentiate the complexity of pedagogical interventions of the Physical Education Teacher. This indicates that the competent initial training should help to recognize and adapt vocational action, identifying variables and concepts involved in each conceptual space and that these spaces should not be confused with physical ones. 


\section{Introducción}

El análisis que se plantea a continuación versa sobre un asunto, una propuesta pedagógica, que tuvo su génesis a principios de siglo, cuando el autor Jorge Pérez Gallardo desarrolló, junto a otros autores, en su libro Educaçao Física Escolar: do berçario ao ensino medio, (Pérez Gallardo, et. al. 2003), y luego también en el libro Gimnasia Rítmica Formativa: una propuesta para la educación física escolar ( Pérez Gallardo \& Linzmayer Gutiérrez, 2013), la problemática que enfrenta el profesor de Educación Física cuando debe desarrollar su intervención pedagógica en los distintos escenarios educativos. Y aunque es evidente que el profesor de Educación Física desarrolla su práctica docente en múltiples escenarios, los espacios conceptuales a los que se hace referencia aquí, superan el simple hecho de un espacio físico. En su origen, fueron descritos como "espacios de actuación profesional del profesor de educación física”, haciendo referencia a ámbitos en los cuales el profesor del área se desenvuelve como profesional. Sin embargo, en más de una oportunidad, cuando se han planteado tales conceptos en clases, congresos, seminarios etc., ocurre la particularidad, producto quizá del imaginario social que se ha creado en torno al área, que muchos piensan que se hace referencia a espacios físicos, (gimnasios, salas de clases, patios, pistas de atletismo, clubes, o jardines infantiles, etc.); en cierta forma puede ser así, pero en realidad es mucho más que eso. Si bien, toda intervención profesional ocurre en un espacio físico, de lo que aquí se trata es distinguir estos espacios desde los conceptos que involucran las prácticas que ocurren al interior de dichos espacios, de ahí la justificación del presente artículo de presentar tales discusiones como "espacios conceptuales", pues se trata de ubicar dicha actuación profesional con conceptos que implican cambios o adaptaciones metodológicas, procedimentales, actitudinales, cognitivas y emocionales. El mismo profesor, modifica o debiera modificar su comportamiento en función de tales espacios conceptuales, pues no es lo mismo intervenir en una sala de clases que en una actividad extraescolar o en un club de entrenamientos.

Siguiendo la reflexión anterior, el presente texto busca distinguir un espacio físico de un espacio conceptual, ya que el espacio físico podría ser el mismo, pero, al cambiar la acción, más precisamente la intencionalidad de la acción, lo que cambia son los conceptos, principalmente pensando en hacer referencia a lo que el profesor "hace" en dichos espacios ya que ese "hacer" está sujeto a objetivos, didácticas, principios y valores humanos de convivencia específicos, esto es, conceptos que implicarán comportamientos diferentes. Esto significa que el profesor adquiere roles diferentes en circunstancias diferentes y que sus acciones y actitudes no son las mismas en espacios-momentos distintos. Puede que esta modificación en el hacer también implique una modificación en el “deber ser”, puesto que cada espacio conceptual obligará al profesional a meditar sobre asuntos éticos y morales que le exigirán una postura frente al fenómeno.

Por otra parte, un espacio físico es dimensionable físicamente, es decir, medible y pueden describirse sus características materiales sin mayores problemas. En cambio, un concepto es una representación mental de un objeto, hecho, cualidad, situación, palabra o forma en que se expresan ideas o conocimientos (RAE, 2014) y, de acuerdo con ello, las representaciones de estos espacios conceptuales implican hechos y situaciones que obligan al profesor a actuar en consecuencia con lo que se espera de él en razón del espacio conceptual en el que se encuentra interviniendo. Lo anterior dice también relación con lo que expresa Berger y Luckmann (1986), en su libro La Construcción Social de la Realidad puesto que las acciones y objetivos que plantean los diferentes espacios corresponden a realidades construidas socialmente, y representan los intereses de quienes participan de tales realidades.

El profesor de Educación Física, por las características de su profesión, se ve involucrado tanto en sala de clases como fuera de ella en forma permanente y sistemática, y, más aun, estas dinámicas profesionales se dan, muchas veces, en el mismo espacio físico. Así como hace clases desarrollando el currículo correspondiente a su especialidad en el gimnasio o patio del colegio, en el mismo lugar debe desarrollar prácticas deportivas o artísticas vinculadas a actividades extra programáticas, y también, a veces, actividades de entrenamientos específicos de algún deporte determinado. Se enfrenta a la complejidad que implica la aplicación de saberes y conocimientos adquiridos durante su formación inicial docente, pues no se trata de utilizar las mismas metodologías de la misma manera en todos los espacios donde trabaja, y se verá interpelado por circunstancias, contextos y personas muy diferentes unos de otros. 
Dicho profesional, por lo general, además, recibe en su formación inicial docente una alta carga técnica en los saberes que debe desarrollar, esto significa que los dominios técnicos adquieren mayor importancia que los dominios conceptuales, lo cual reduce dicha formación a un nivel instruccional, que determina gran parte de su praxis pedagógica cuando debe intervenir en el medio escolar.

Por el contrario, lo que corresponde en la formación inicial docente es el desarrollo del dominio conceptual, pues con tales dominios podemos analizar, comprender y explicar los fenómenos referidos a tales ámbitos.

La universidad es uno de los lugares espacio/tiempo, donde se deben generar nuevas ideas, pero estas solo son posibles si las dinámicas relacionales humanas que ocurren en su interior establecen diálogos que van más allá de las ejecuciones técnicas. Si un estudiante, futuro profesor de Educación Física, dedica la mayor parte del tiempo de su formación inicial docente a la ejecución de técnicas deportivas, y, además, solo es evaluado en la forma como ejecuta tales técnicas, lo más probable es que crea que eso es lo que se espera de él en el medio donde desarrollará su práctica pedagógica. Pues bien, si su formación ocurre en una universidad esto debe ser exactamente todo lo contrario. Es decir, los énfasis deben ser conceptuales, no técnicos. De ahí que se deba insistir en que no es lo mismo espacios conceptuales que espacios físicos.

Por otra parte, el dominio conceptual implica no solo un conocimiento técnico, sino además y preferentemente, la capacidad de explicar fenómenos que trascienden la especialidad, creando relaciones entre conceptos aparentemente diferentes, permitiendo la comprensión de por qué, para qué y cómo suceden los hechos socioculturales, captando la simbiosis que ocurre entre elementos de la naturaleza y la cultura. En definitiva, el dominio conceptual permite explicar y comprender el comportamiento humano individual y social, y, en nuestro caso, saber que no es lo mismo intervenir en el espacio de vivencia que en el espacio de práctica o en el de entrenamiento. Juntamente con ello, el dominio conceptual permite descifrar los códigos simbólicos que permanecen ocultos en las dinámicas relacionales humanas a la luz de las interacciones sociales, especialmente las deportivas. Al respecto se sugiere la lectura del texto Educación Física y Aprendizaje Social del autor Valter Bracht (1992).

Generalmente, en la enseñanza de los deportes en las escuelas se enfatiza el respeto incondicional e irreflexivo a las reglas, y da a estas un carácter estático e incuestionable, lo que no lleva a la reflexión y al cuestionamiento, esto es, forja un ser humano que no cuestiona y no se opone a lo que se le impone. Por ello, es conveniente que observemos que si bien, el profesor de Educación Física debe desarrollar habilidades técnicas, tan importante como ello es el desarrollo de un dominio conceptual que le permita analizar, criticar y traducir códigos de dominio cultural inmersos en las practicas corporales. Lo anterior justifica una formación académica que debe darse en la universidad, de tal manera que permita al profesor de Educación Física superar la práctica por la práctica, en definitiva, reconocer su responsabilidad ética más allá de una responsabilidad técnica, lo que se traduce en la aplicación del principio pedagógico de acción-reflexión-acción.

Siendo así, es necesario que el profesional de Educación Física, reconozca que una de sus primeras responsabilidades es contextualizar, es decir, antes de iniciar su intervención reconocer el espacio en el cual desarrollará su práctica, de tal manera que las personas que están a su cargo pueden sacar el mejor provecho posible del momento que comparten junto a su profesor. Una vez que haya contextualizado, buscará flexibilizar y, posteriormente, problematizar en función de los objetivos que se hayan establecido para cada uno de los espacios conceptuales para los cuales se ha formado. Esto es, un profesional formado competentemente será capaz de reconocer los conceptos que representan el proceso de enseñanza aprendizaje tanto en la clase como en las actividades extraescolares, deduciendo que el entrenamiento implica conceptos que, muchas veces, escapan a sus dominios, pues este considera saberes más específicos.

En resumen, es necesario considerar que los dominios técnicos no debieran ser prioridad de una formación inicial docente en la universidad, y que los espacios conceptuales que a continuación se indican ayudarán al profesor a contextualizar su intervención profesional. 


\section{El espacio conceptual de Vivencia}

Se entiende por vivencia "las experiencias corporales que el estudiante tiene en el o los momentos de cada clase de Educación Física y que ocurren en el ámbito escolar, su objetivo es poner a los estudiantes en contacto con la cultura corporal, partiendo del presupuesto de que la cultura es un patrimonio universal a la cual todo ser humano debería tener derecho”, (Pérez Gallardo \& Linzmayer Gutiérrez, 2013 p.16).

Una de las principales características de este espacio es el poco tiempo que se le asigna para que los profesores del área puedan lograr algún aprendizaje, puesto que todo lo que se enseña en una clase, especialmente si se refiere a dominios técnicos, se olvida al día siguiente; y, como hay que esperar hasta la próxima semana, francamente no tiene sentido repetir la misma actividad. Dentro de las limitaciones del ambiente escolar (un máximo de dos horas semanales), y, considerando la enorme cantidad de contenidos a ser ofrecidos a los estudiantes, es necesario una organización y una planificación de esos contenidos, de forma que en cada nivel los alumnos tomen contacto con aquellos contenidos que por las características de desarrollo (necesidades, expectativas e intereses) sean más adecuados para ellos.

Aquí, entra en juego otro concepto: tiempo. ¿Cuánto tiempo tienen los niños y niñas en una clase de Educación Física en la escuela para aprender determinado contenido, especialmente en sus aspectos técnicos?, no más de 45 minutos. Ahora bien, si se parte de la base que la función de la escuela no es preparar técnicos, entonces no se justifica buscar tales aprendizajes, menos en Educación Física, pues no son los aspectos técnicos los que las personas usan para desenvolverse en su vida cotidiana, entendiendo por gesto técnico movimientos y actitudes relativos a los deportes, gimnasias o danzas, etc. La función básica, primaria y fundamental de la escuela, es preparar ciudadanos para la vida y la sociedad que representarán, utilizando para ello diversos saberes del conocimiento, en los cuales se enfatice valores y principios de convivencia humana deseables por una sociedad determinada.

Los autores indican que, en este espacio, "el interés pedagógico no está centrado en el dominio técnico de los contenidos, pero si en el dominio conceptual (en la perspectiva de un análisis histórico-crítico que supere el sentido común), dentro de un espacio humano de convivencia, donde puedan ser vivenciados aquellos valores humanos que aumenten los grados de confianza y de respeto entre los integrantes del grupo” (Id. p.16). Es decir, en este espacio, en la clase, es donde el profesor debe explicar para buscar un razonamiento crítico de lo que se está enseñando, considerando obviamente el nivel del curso puesto que no es lo mismo un primero básico que un primerio medio. Además, se debe considerar la enorme cantidad de contenidos de la cultura corporal al cual el alumno tiene derecho a conocer, o por lo menos saber que existen. De ahí que se llama la atención sobre no dar preferencia de algunos contenidos sobre otros, especialmente considerando que "no existe una práctica corporal mejor o peor, de la misma forma como no existen juegos, danzas, luchas, deportes o gimnasias que todos deban saber” (Neira, 2010).

Las clases deben seguir una planificación para posibilitar la vivencia de todas las manifestaciones culturales que son parte de la Educación Física, obteniendo un dominio conceptual que faciliten su transferencia para varias otras situaciones o contextos. La variedad de contenidos ofrecidos aumenta las posibilidades de elección de los alumnos para la formación de grupos de práctica que podrán desarrollarse con mayor profundidad en el espacio de práctica (extraescolar).

En este espacio conceptual de vivencia, que representa todo lo que ocurre en la clase de Educación Física regular dentro de las escuelas y liceos, el profesor debe ofrecer a sus estudiantes todo el conocimiento universalmente producido relativo a las manifestaciones de la cultura corporal o practicas corporales que se representan a través de juegos, deportes, gimnasias, luchas, danzas, actividades en el medio natural, elementos de las artes escénicas, musicales y plásticas). Como se comprenderá esto representa un extenso acervo de conocimiento por lo cual resulta imposible, con el poco tiempo que se cuenta en la clase, preocuparse con dominios técnicos. Además, para cualquier profesor de Educación Física dominar las técnicas de cada uno de dichos contenidos resultaría prácticamente imposible. Pero lo que no parece imposible es el dominio conceptual que debiera tener al respecto 
de tales fenómenos, pues su deber pedagógico es, precisamente, explicar por qué esos fenómenos ocurren de la forma que ocurren, buscando junto a sus estudiantes los sentidos y significados que tales prácticas tienen para determinados grupos humanos, lo que implica investigar tales fenómenos junto a sus alumnos antes de desarrollarlos en clases. También es necesario realizar una ficha de las actividades o practicas corporales de todos y cada uno de los estudiantes, de lo que ellos ejecutan cuando no están en la escuela y que son de uso cotidiano La responsabilidad del profesor en este espacio tiene que ver con, primero: poner en contacto a sus estudiantes con todas las manifestaciones de la cultura corporal producida por los seres humanos y, segundo: analizar, interpretar y re-significar, permitiendo que se creen nuevas formas de ejecutarlos (Neira, 2010). Se debe recordar que la función fundamental de la escuela es la formación de la ciudadanía, del derecho y deber cívico, de personas conscientes de sus realidades socioculturales y asumidas en su compromiso con la democracia y libertades individuales. Por ello la cooperación se transforma en el eje central de las acciones y evitando las competiciones que dividen y separan. Por tanto, no debe ocuparse la clase en actividades de preparación física o entrenamiento de cualidades deportivas, pues no se cuenta ni con el tiempo ni las condiciones materiales para desarrollar sistemas físico-orgánicos de los individuos, al contrario, este espacio debe llevarnos a la reflexión de nuevas posibilidades de pensamiento y acción, reflejando una educación de seres humanos libres y creadores, apoyado en acciones cooperativas no competitivas.

\section{El espacio conceptual de Práctica}

Según Pérez Gallardo et.al. (2003) (p. 19), “es un espacio de libre organización de los estudiantes, donde escogen las manifestaciones de la cultura corporal que fueron vistos en la clase de Educación Física (vivencia) y que despertaron mayor interés en ellos (más relevantes para ellos), creando así grupos de interés”. El objetivo en este espacio es lograr que los alumnos aprendan a dominar y estabilizar técnicas de ejecución de los contenidos escogidos. Aquí aumenta el tiempo de experimentación y existe una mayor preocupación con la técnica (Capacitación), pues ejecutando correctamente, el alumno tiene más posibilidades de interactuar con su grupo. Este es el espacio de las actividades extra-escolares y comunitarias, las que deberían ser organizadas y administradas por los propios integrantes del grupo social y coordinados por el profesor de Educación Física. Este espacio, para que mantenga su carácter pedagógico, debe nacer de las vivencias de clase y para que eso ocurra el profesor debe diversificar los contenidos, pues de no ser así, los estudiantes no podrán tener acceso ni podrán saber si tienen o no habilidades para nuevas prácticas corporales.

Generalmente, las actividades extraescolares son ofrecidas en las escuelas en la medida que se cuenta con especialistas de determinadas prácticas corporales, y si tal especialista no existe, la escuela no la ofrece.

Es aquí donde aparece una figura importante: la co-responsabilidad de la enseñanza. Esta figura dice relación con la posibilidad que los estudiantes tienen de ofrecer a sus compañeros los conocimientos respectos de lo que cada uno práctica, compartiendo sus habilidades y transformándose en líderes de tales prácticas, creando grupos de interés que representarán al colegio ante la comunidad, favoreciendo la extensión cultural de la escuela. Aquí el profesor es coordinador/orientador, participa lateralmente, pues la actividad es dirigida por los estudiantes, contribuyendo así a la formación de líderes comunitarios, al desarrollar en ellos la capacidad de organizar, administrar y definir sus propios objetivos y actividades. Es el espacio ideal para el desarrollo de la autonomía.

\section{El espacio conceptual de Entrenamiento}

En este espacio los conceptos hacen referencia al alto rendimiento deportivo, cursos de especialización, periodización, talento y habilidades técnicas específicas, técnico deportivo, etc. Aquí estamos en la depuración de movimiento, y la preparación física y mental de deportista en la obtención de un objetivo claro y preciso: ser campeón en su especialidad deportiva.

El objetivo en este espacio conceptual de actuación profesional, es lograr que el individuo internalice las técnicas de una modalidad especifica de la cultura corporal. Para ello, se necesita una cantidad mayor de tiempo, con 
materiales específicos y una disposición particular (biológica y sicológica) para tener algún grado de éxito en la modalidad elegida. Además del material específico, este espacio necesita de un profesor o técnico con conocimientos avanzados, pues el objetivo principal es la ejecución correcta de las habilidades que forman parte de las manifestaciones culturales competitivas, para que el educando o atleta pueda participar con éxito en eventos deportivos competitivos. Este espacio corresponde a los clubes o instituciones que se dedican a actividades de alto rendimiento (deportes o espectáculos artísticos). Todo esto implica una programación seria y sostenida en el tiempo, en la cual el profesor-entrenador visualiza cuales son los momentos en los que el atleta se encontrará en su mejor forma deportiva para competir. Aquí el dominio técnico pasa a ser más importante que el dominio conceptual, sin embargo, se supone que el profesor/entrenador domina los conceptos que involucra una preparación deportiva a este nivel. Es un espacio en el cual la practica repetitiva de los movimientos es lo que le dará sentido al entrenamiento, pues no se llegará a la meta analizando y criticando conceptos socioculturales, sino tan solo mediante un exhaustivo periodo de entrenamiento que permita alcanzar el máximo desarrollo del potencial biológico, físico y orgánico del atleta. Para que esto sea posible, es necesario, además, la concurrencia de políticas públicas que apoyen con infraestructura y dinero para la ejecución de las diferentes etapas del alto rendimiento (Periodización del Entrenamiento -mejoría de la condición física y técnica, campeonatos regionales, nacionales e internacionales, etc.).

Cabe decir finalmente que la formación inicial docente del profesor de Educación Física debe asegurar el desarrollo de las competencias técnicas y conceptuales que le permitan distinguir los conceptos descritos en este estudio y, considerando los contextos, actúe en consecuencia.

\section{Cuadro 1: Resumen Espacios Conceptuales de la Actuación Profesional del Profesor de Educación Física}

\begin{tabular}{|c|c|c|c|c|c|c|}
\hline $\begin{array}{l}\text { Variable } \\
\text { Espacio }\end{array}$ & Finalidad & $\begin{array}{l}\text { Dominio de } \\
\text { Aprendizaje }\end{array}$ & Tiempo & Lugar & $\begin{array}{l}\text { Énfasis ético/ } \\
\text { pedagógico }\end{array}$ & $\begin{array}{l}\text { El alumno } \\
\text { Aprende a }\end{array}$ \\
\hline Vivencia & $\begin{array}{l}\text { Contacto con las } \\
\text { diferentes } \\
\text { manifestaciones } \\
\text { de la cultura } \\
\text { corporal }\end{array}$ & Conceptual & $\begin{array}{c}2 \text { horas a } \\
\text { la } \\
\text { semana }\end{array}$ & $\begin{array}{l}\text { Clase de } \\
\text { Educación } \\
\text { Física }\end{array}$ & $\begin{array}{c}\text { Formación } \\
\text { Humana }\end{array}$ & $\begin{array}{l}\text { Conocer y valorar } \\
\text { su cultura corporal } \\
\text { Patrimonial. }\end{array}$ \\
\hline Práctica & $\begin{array}{c}\text { Libre } \\
\text { organización de } \\
\text { los alumnos } \\
\text { (autonomía) }\end{array}$ & Técnico & $\begin{array}{c}4 \text { a } 5 \\
\text { horas a } \\
\text { la } \\
\text { semana }\end{array}$ & Extra-escolar & $\begin{array}{c}\text { Formación } \\
\text { Humana y } \\
\text { Capacitación }\end{array}$ & $\begin{array}{l}\text { Dominar técnicas / } \\
\text { Representación } \\
\text { comunitaria }\end{array}$ \\
\hline Entrenamiento & $\begin{array}{l}\text { Internalizar } \\
\text { (automatizar) } \\
\text { técnicas } \\
\text { específicas del } \\
\text { deporte }\end{array}$ & $\begin{array}{l}\text { Rendimiento } \\
\text { deportivo }\end{array}$ & $\begin{array}{c}5 \text { a } 8 \\
\text { horas } \\
\text { diarias } \\
\text { más de } 5 \\
\text { veces } \\
\text { por } \\
\text { semana }\end{array}$ & $\begin{array}{l}\text { Clubes de alto } \\
\text { rendimiento }\end{array}$ & Capacitación & Competir \\
\hline
\end{tabular}

Prof. Dr. Luis Linzmayer Gutierrez (2016) 


\section{Bibliografía}

Berger, p.; Luckmann, t. (1986). La construcción social de la realidad. Buenos Aires, Argentina: amorrortu.

Pérez Gallardo, J. (organizador) (2003) Educação Física Escolar: do berçário ao ensino médio. Brasil. Lucerna.

Pérez Gallardo, J \& Linzmayer Gutiérrez, L. (2013) Gimnasia Rítmica Formativa: propuesta pedagógica para la educación física escolar. Santiago de Chile. Chile. Universidad del Bio-Bio.

RAE. (2014) Real Academia Española. Diccionario de La Real Lengua Española. 23a Edición del Tricentenario.

Bracht, V. (1992) Educación Física y Aprendizaje Social. Argentina: Vélez Sarsfield.

Garcia Neira, M. (2010). El Currículo de Educación Física en la Perspectiva Cultural: fundamentos y práctica pedagógica. Horizontes Educacionales 15, (2) pp. 49-67. Recuperado de: http://www.redalyc.org/articulo.oa? $\underline{\mathrm{id}=97917578004}$ 